\title{
Adolescentes que utilizan servicios de atención primaria: ¿Cómo viven? ¿Por qué buscan ayuda y cómo se expresan?
}

\author{
Adolescent clients of primary health care services. \\ How do they live? Why do they seek help, \\ and how do they express themselves?
}

Lílian dos Santos Palazzo 1,2

Jorge Umberto Béria 3

Elaine Tomasi 4,2

\footnotetext{
1 Pós-Graduação em Saúde Coletiva, Universidade Luterana do Brasil. Rua Miguel Tostes 101, Canoas, RS 98420-280, Brasil. Ispalazzo@bol.com.br 2 Secretaria Municipal de Saúde e Bem-estar. Rua Lobo da Costa 1764, Pel otas, RS, Brasil. 3 Pós-Graduação em Medicina, Universidade Luterana do Brasil. Rua Miguel Tostes 101, Canoas, RS 98420-280, Brasil. jberia@terra.com.br 4 Centro de Pesquisas Epidemiológicas, Universidade Federal de Pelotas. C. P. 464 Pel otas, RS 96001-970, Brasil. etomasi @terra.com.br
}

Abstract The objective of this study was to characterize adolescent clients of community-based primary health care services in order to understand: 1) how they express themsel ves when they seek medical hel p; 2) the health care they receive; and 3) key social and family factors. Among 35 urban primary care services belonging to the municipal public health department in Pelotas, Rio Grande do Sul State, Brazil, 10 were randomly sel ected, and all individuals between 13 and 19 years of age who had previously had an appointment with a non-psychiatric physician were interviewed during a three-month period $(n=463)$. U se of the services has more of a more curative than preventive focus. Males seek medical help less frequently and mainly due to physical complaints. Females tend to express themsel ves on the basis of sexual problems. Few adol escents expressed their emotional problems, although some mentioned family and school problems as well as stressful situations.

Key words Teen Health; Primary Health Care; Sexuality; Health Services

Resumen El objetivo del estudio fue caracterizar a los adolescentes que utilizan los servi ci os de atención primaria de una comunidad; saber cómo se expresan en la búsqueda de ayuda médica, la atención recibi da y aspectos soci ofamiliares. De entre un total de 35 unidades de atención primaria urbanas pertenecientes a la red de salud pública del municipio de Pelotas (Brasil), 10 fueron el egidas al azar y entrevistados todos los indi viduos entre 13 y 19 años que consultaron al médico y no al psiquiatra durante tres meses. ( $n=463$ ). La utilización de los servicios tiene un carácter más bien curativo que preventivo. Los varones acuden menos y lo hacen principalmente con quejas somáticas, mientras que las jóvenes suelen manifestar quejas sexuales. Raros son los que expresan sus aflicciones emocional es, aunque al gunos relatan problemas familiares, escolares y situaciones estresantes.

Palabras clave Salud delos Adolescentes; Atención Primaria; Sexualidad; Servici os de Salud 


\section{Introducción}

La etapa de la adolescencia tiene una importancia vital, pues es cuando se produce la estructuración de la personalidad del individuo, a través de intensos cambios en los aspectos corporales, psicológicos y de relación. Es cuando el sujeto experimenta los mejores índices de salud y vitalidad que le permitirán Ilevar a cabo las tareas de la edad adulta. Sin embargo, esta capacidad vital se halla afectada en un número creciente de jóvenes, debido a problemas como: violencia física, abandono, suicidio, abuso de substancias psicoactivas, enfermedades sexualmente transmisibles, trastornos mentales, evasión escolar, problemas alimentarios, embarazo precoz, entre otros (Curbelo, 1987; Rubio, 1987; Walker \& Townsend, 1998; WHO, 1999).

En Brasil, según el Ministerio de Salud (MS, 2003a), un $21,84 \%$ de la población del país está formada por jóvenes con edad entre 10 y 19 años. La primera causa de mortalidad son los problemas denominados "externos", o sea, accidentes de tránsito, homicidios y suicidios. Un 45,9\% de las jóvenes con edad entre 15 y 19 años sexualmente activas no utilizan ningún método anticonceptivo; Ios casos de SIDA en jóvenes con edad comprendida entre 10 y 19 años es de un 2,5\% del total de casos. En la ciudad de Pelotas, Rio Grande do Sul, Brasil, donde este estudio fue realizado, la prevalencia de depresión en adolescentes que consultan en la atención primaria es de un 26, 6\% (Palazzo, et al., 2001); el uso frecuente de alcohol entre escolares es de un $16,8 \%$ y el tabaco aparece en un $11,6 \%$ (Tavares et al., 2001). Estos problemas son preocupantes, pues interfieren en diferentes grados en el desarrollo de los adolescentes para que se transformen en sujetos productivos y socialmente activos.

Además, en la adolescencia, los intensos cambios vividos y la búsqueda de identidad contribuyen a que muchas veces el joven se vea perdido entre una multitud de estímulos internos y externos, entre varias y nuevas alternativas por las que tiene que optar, necesitando ayuda de los adultos. No obstante, muy a menudo sus mensajes y demandas de ayuda, comprensión y orientación, vienen enmascaradas por una conducta agresiva o de introversión, o incluso otras de otra índole, que despiertan respuestas en su entorno también hostiles o de desatención. Esta dificultad de comunicación entre adultos y jóvenes también puede verse reflejada en la atención prestada a los adolescentes, que a su vez provoca que las principales causas de mortalidad y morbilidad en esa etapa - potencialmente prevenibles - pasen a no recibir la asistencia debida (Blum, 1987; Marks et al., 1990; Ziv et al., 1999).

Así, el objetivo de este estudio es caracterizar la demanda adolescente de las unidades de atención primaria de la ciudad de Pelotas; conocer los motivos por los que ellos acuden a la consulta médica, cómo se expresan y la atención que reciben. Asimismo, se buscó presentar una noción sobre el contexto en el que se desarrolla el joven, pues la comprensión de cualquier problema adolescente nos Ileva siempre al estudio de con qué adultos está en relación y de qué tipo de estructura social y familiar se genera.

Hemos el egido como ámbito de estudio el de la atención primaria, porque en el sistema de salud pública de Brasil y en muchos países, los centros de atención primaria desempeñan la función de "puerta de entrada". Asimismo, el Ministerio de Salud Brasileño (MS), desde 1989, desarrolla un programa titulado Programa de Saúde do Adolescente (PROSAD - MS, 2003a) que tiene como objetivo la promoción de la salud integral del joven para favorecer el proceso general de su crecimiento y desarrollo, buscando reducir la morbilidad, los desajustes individuales y sociales. Sin embargo, en nuestro país son escasos los estudios sobre la utilización de los servicios de atención primaria por adolescentes. No obstante, se reconoce en la literatura mundial, que los jóvenes casi no buscan estos servicios (Klein et al., 1998; Ziv et al., 1999). Así, este estudio pretende contribuir para futuros programas de capacitación del profesional sanitario, profesores y líderes comunitarios, además de aportar datos que puedan apoyar las actividades del Programa de Salud de la Familia (PSF) y Programa de Agentes Comunitarios de Salud (PACS) del MS (2003b) para una efectiva promoción y prevención en la salud del adolescente y su familia.

\section{Metodología}

Es un estudio descriptivo basado en servicios públicos de atención primaria de salud y es parte de una investigación sobre depresión en la adolescencia (Palazzo et al., 2001). Los servicios forman parte de la red pública de atención de la ciudad de Pelotas que consta de 35 centros o unidades de atención primaria localizadas en la zona urbana. Toda la salud pública del municipio está bajo la coordinación y gestión de la Secretaría Municipal de Salud (SMSBE) y, hasta el momento de la realización del estudio, no existían equipos de PSF y el PACS estaba en 
fase inicial de organización. Para ese estudio fueron elegidas al azar diez unidades y fueron incluidos todos los individuos con edad entre 13 y 19 años que acudieron a estos servicios para consultar con un médico general, ginecólogo o pediatra.

Para calcular el tamaño de la muestra se utilizó la metodología para estudio de prevalencia (Epi Info) considerando: (a) número aproximado de jóvenes que van mensualmente a la consulta en las unidades, según la SMSBE: 2000; (b) frecuencia esperada para depresión en atención primaria: $30 \%$; (c) nivel de confiabilidad: 95\%; (d) posibles pérdidas: 10\%; (e) precisión: $5 \%$. El resultado fue 306 adolescentes. Durante el período comprendido entre octubre del 1997 y enero del 1998 se presentaron a las unidades 497 jóvenes. No fueron entrevistados 26 principalmente por rehusarse a participar del estudio (11) o por falta de permiso (15). De las 471 entrevistas realizadas, 8 fueron excluidas ( 5 por duda en relación con la calidad de las respuestas y 3 por inclusión equivocada). Así, hemos tenido 34 pérdidas $(6,8 \%)$ y la muestra total del estudio quedó en 463 adolescentes.

Variables estudiadas e instrumento utilizado

- Sociodemográficas: sexo, edad, color, renta familiar per cápita, estado civil del entrevistado, actividad actual y escolaridad.

- La consulta médica: motivo principal de la búsqueda de atención, asunto que al adolescente le gustaría haber conversado con el médico y no fue posible, exámenes solicitados, prescripción de medicinas, y existencia de consulta anterior. El motivo principal que propició la búsqueda de atención fue evaluado a través de una cuestión de tipo abierto. Se buscó transcribir la forma exacta como se expresó el adolescente y, posteriormente, estos motivos fueron reunidos en veinte grupos según criterio de homogeneidad, con el objetivo de permitir el análisis cuantitativo de estos datos. De manera semejante, hemos procedido con la variable asuntos, que fueron reunidos en cinco grupos. El diagnóstico médico (CIE - 10) se consideraron los dos que aparecen en la Ficha de Atención Ambulatoria (FAA) del Sistema de Información Ambulatoria - Sistema Único de Salud (SIA - SUS) padronizado por el Ministerio de Salud brasileño. La pregunta sobre la prescripción de medicinas admitía dos alternativas y ambas fueron agrupadas según la "International Classification of Process in Primary Care IC-Process-PC " (WONCA, 1986).

- Estructura y relaciones familiares: número de personas que viven en la casa, presencia de hermanastro que vive en el mismo hogar, actividad y escolaridad de los padres, abuso de alcohol y/ o drogas por algún familiar que vive en el hogar. Investigamos solamente a los adolescentes que viven con ambos padres o por lo menos con uno de ellos ( $n=330 ; 71,3 \%$ del total de la muestra), la forma en la que los padres reaccionan cuando el hijo hace algo indebido, el modo como recompensan cuando hace algo que les parece correcto, el criticismo de los padres, la colocación de algunos límites por parte de los mismos y el interés de los padres por los estudios de los hijos.

- Abuso de alcohol y/o drogas ilícitas por el entrevistado;

- La ocurrencia de situaciones de estrés y como éstas le han afectado. Para responder a las preguntas de este bloque, el entrevistado recibió una tarjeta con las alternativas para que eligiera una de ellas: "no ocurrió”, “ocurrió y no me afectó”, “ocurrió y me afectó algo”, “ocurrió me afectó mucho", y “ocurrió me alegró”. Las variables estudiadas fueron: muerte del padre, muerte de la madre, muerte de hermano, muerte de otro ser querido, adopción, dejar los estudios, cuidar de hermanos o personas mayores, dificultades materiales, separación o divorcio de los padres, violencia física, bajo rendimiento en la escuela o en el trabajo, suspensión en la escuela, conflictos continuos interpersonales, empezar a trabajar, enfermedad grave en familiar que vive en el mismo hogar, enfermedad grave en otro pariente o amigo, pasó a vivir sin la familia, dificultades de relación en la escuela o trabajo, carencia de un confidente, cambio de ciudad, escuela o barrio.

El instrumento utilizado fue una entrevista semi-estructurada construida para responder a los objetivos del estudio.

\section{Sistemática de recogida de los datos}

Los entrevistadores fueron entrenados a diario durante dos semanas y el estudio piloto se desarrolló durante una semana en cinco unidades. La recogida de los datos fue realizada con un entrevistador en cada turno, todos los días, en todos los centros elegidos y el cuestionario se realizaba con el adolescente inmediatamente después de la consulta médica. El control de calidad fue llevado a cabo a través de reuniones semanales con todos los entrevistadores y la coordinadora del estudio, revisión de cada una de las entrevistas para posterior discusión sobre el trabajo, comparación entre la numeración de los cuestionarios entregados a los entrevistadores y los devueltos, llamadas y visita sorpresa a las unidades. 
Análisis de los datos

Los datos fueron digitados por dos auxiliares del estudio, en dos archivos distintos que fueron posteriormente comparados utilizándose el paquete estadístico Epi Info, con el objetivo de evitar sesgos en esa fase. EI SPSS/PC+fue utilizado para medir la frecuencia de la distribución de las variables y también para la verificación de las asociaciones entre las variables básicamente a través del Test del Chi-Cuadrado $\left(\chi^{2}\right)$ y del Coeficiente de Correlación de Pearson.

\section{Resultados}

Los adolescentes que buscan los centros de atención primaria municipales pertenecen principalmente al sexo femenino (79,3\%). El sexo femenino sigue siendo el más prevaleciente, incluso cuando se las excluye por consultar por motivos relacionados con el embarazo, con una proporción de cerca de un 3.1 respecto al sexo masculino.

En cuanto a la edad, un 42,5\% están en la franja de 15 a 17 años, con un promedio de 16 años. La mayoría de los jóvenes es de color blanco $(77,5 \%)$ y solteros $(73,7 \%)$. No fueron encontrados analfabetos, aunque un 10,8\% tenga entre 1 y 3 años de escolaridad y muchos tienen entre 4 y 6 años de escolaridad (41,8\%). La renta per cápita de la familia está principalmente entre $1 / 2$ a 1 sueldo mínimo (34,9\%), con un promedio de 1 sueldo mínimo per cápita (Tabla 1).

Se observa que los adolescentes más jóvenes (13-14 años) acuden a las unidades incentivados principalmente por la familia $(66,7 \%)$, mientras los mayores (18-19 años) lo hacen más por iniciativa propia (56,3\%). Un $56,7 \%$ ha consultado al médico general, un $39,8 \%$ al ginecólogo y un 3,5\% al pediatra. A medida que el adolescente se va desarrollando va aumentando la búsqueda de atención $(p<0,05)$. También se observa que, a medida que va aumentando la edad, aumenta la utilización de este servicio por parte de las mujeres $(p<0,03)$.

La mayoría busca la ayuda de los médicos principalmente a través de quejas (Tabla 2) dispersas en el ámbito somático $(61,1 \%)$ o vinculadas a la sexualidad (37,4\%), estas últimas así dispuestas: embarazo (24,6\%), menstruación con o sin otros síntomas (3,9\%), relacionada con los genitales $(3,9 \%)$, relaciones sexuales con y/ o anticonceptivos ( $3,7 \%)$, y relativo a las mamas (1,3\%). No obstante, cuando se estratificó por sexo, fue verificado que los motivos de consulta más frecuentes entre los varones son
Ios relacionados con el aparato respiratorio $(21,9 \%)$, quejas dermatológicas $(20,8 \%)$ y relacionados con el sistema musculoesquelético $(13,5 \%)$. Entre las chicas, los motivos más frecuentes son los relacionados con el embarazo (31\%), al aparato respiratorio (7,7\%) y el sistema musculoesquelético (6,8\%). Llama la atención que las relaciones sexuales o anticoncepción fueron relatadas como motivo de consulta por 17 adolescentes $(9,8 \%)$ y todos pertenecientes al sexo femenino. Se observa, también que los motivos relacionados con la sexualidad van aumentando según aumenta la edad ( $p<0,005)$.

Cabe destacar que tan sólo 7 adolescentes $(1,5 \%)$ presentaron quejas vinculadas al campo psicosocial. También Ilama la atención que sólo 31 jóvenes (6,7\%) afirmaron que les hubiera gustado hablar acerca de otro tema en la consulta. Sin embargo, para nueve de estos casos no les fue posible hablar por falta de tiempo u oportunidad; para siete, la imposibilidad fue por inhibición o vergüenza suya; para dos, fue por las dos razones; nueve se olvidaron de hablar sobre ello; dos han contestado que el asunto no tenía importancia; uno relató que no conversó por miedo de causar problemas en casa; y uno creía que el asunto era tema para el médico general.

Se considera importante poner en relevancia que el consumo de drogas no aparece como motivo de consulta y, cuando es preguntado en la entrevista, el informe de uso de drogas ilegales fue inexpresivo. Sin embargo, el alcohol fue mencionado como la droga más utilizada por los adolescentes $(34,5 \%)$ principalmente los del sexo masculino ( $p<0,05)$ consumido principalmente una vez a la semana (48,1\%), seguido del tabaco $(27,8 \%)$ con frecuencia de uso a diario $(70,5 \%)$, sin diferencia significativa entre los sexos.

De los 396 adolescentes que ya habían consultado anteriormente, un 78,8\% consultó en el mismo centro de atención primaria y el $21,2 \%$ en otro local. El $56,8 \%$ de estos casos fue por motivo distinto y el $43,2 \%$ fue por el mismo problema. De los 171 adolescentes que consultaron con el mismo médico, un $67,3 \%$ fue por el mismo problema, principalmente embarazo (41\%).

Fueron encaminados a otro profesional 54 adolescentes ( $11,7 \%$ del total de la muestra). El médico ha pedido exámenes para 221 adolescentes $(47,7 \%)$ y los más solicitados fueron las pruebas de laboratorio (68,3\%). Hubo prescripción de medicinas para 255 adolescentes (55,1\% de la muestra). Las más prescritas fueron las del grupo de los antiinfecciosos sistémicos (62/288 veces) seguido de las del grupo de los analgésicos y antiinflamatorios (60/ 288 veces). Los diagnósticos, en su mayoría, están vinculados al plan somático $(61,6 \%)$ y muchos relacionados 
Distribución sociodemográfica de los adolescentes.

Unidades de atención primaria municipales/centro urbano del sur de Brasil, 1997-1998.

\begin{tabular}{|c|c|c|c|c|}
\hline Variables & $\mathrm{n}$ total1 & $\begin{array}{l}\% \text { del total } \\
\text { masculino }\end{array}$ & $\begin{array}{l}\% \text { del total } \\
\text { femenino }\end{array}$ & $\%$ total \\
\hline Edad 2 & 463 & & & \\
\hline $13-14$ & & 31,6 & 18,6 & 21,4 \\
\hline $15-17$ & & 39,8 & 43,3 & 42,5 \\
\hline $18-19$ & & 28,6 & 38,1 & 36,1 \\
\hline Color & 463 & & & \\
\hline Blanco & & 73,5 & 78,6 & 77,5 \\
\hline No blanco & & 26,5 & 21,4 & 22,5 \\
\hline Estado civil2 & 463 & & & \\
\hline Soltero & & 95,9 & 67,7 & 73,7 \\
\hline Casado & & 4,1 & 32,3 & 26,3 \\
\hline Actividad actual2 & 463 & & & \\
\hline Sólo estudia & & 54,1 & 43,6 & 45,8 \\
\hline Sólo trabaja & & 13,3 & 6,6 & 8,0 \\
\hline Estudia y trabaja & & 16,3 & 6,8 & 8,9 \\
\hline $\mathrm{Ni}$ estudia, ni trabaja & & 16,3 & 43,0 & 37,4 \\
\hline Escolaridad (años) & 462 & & & \\
\hline $1-3$ & & 10,2 & 11,0 & 10,8 \\
\hline $4-6$ & & 37,8 & 42,9 & 41,8 \\
\hline $7-8$ & & 32,7 & 27,5 & 28,6 \\
\hline 9 ó más & & 19,4 & 18,7 & 18,8 \\
\hline Renta familiar per cápita & 433 & & & \\
\hline Hasta $1 / 2$ SM & & 27,5 & 27,8 & 27,7 \\
\hline $1 / 2-S M$ & & 37,4 & 34,2 & 34,9 \\
\hline $1-2 \mathrm{SM}$ & & 27,5 & 27,5 & 27,5 \\
\hline más de $2 \mathrm{SM}$ & & 7,7 & 10,5 & 9,9 \\
\hline
\end{tabular}

1 Los totales no coinciden debido a la falta de información sobre algunos adolescentes.

2 Diferencia entre los sexos estadísticamente significativa $(p<0,01)$.

$\mathrm{SM}=$ Sueldo Mínimo.

con el ámbito de la sexualidad $(38,4 \%)$ principalmente el embarazo ( 100 casos $=21,6 \%$ del total de la muestra).

Muchos de los adolescentes viven con ambos padres $(42,1 \%)$ o con el esposo con o sin hijos $(22,7 \%)$. Sin embargo, se puede observar que un $26,3 \%$ dice estar casado o viviendo maritalmente lo que supone que un $3,6 \%$, a pesar de vivir en pareja, sigue probablemente en la casa de los padres. La familia suele estar principalmente constituida por 4 a 6 miembros $(54,7 \%)$ con un promedio de 4 personas. Un $45,6 \%$ poseen entre 1 y 2 hermanos, con un promedio de 2 hermanos.

Los hogares monoparentales totalizan un $20,3 \%$ (un $18,1 \%$ tienen la presencia de la madre y $2,2 \%$ la del padre). Se observa que hubo una reestructuración de la familia en un $6,9 \%$ de los casos, pero sólo 3 adolescentes $(0,6 \%)$ viven con el padre y su nueva compañera. Un $19,7 \%$ de los jóvenes conviven en el mismo hogar con hermanastros. La mayoría de los padres trabajan, sin embargo hay un 3,3\% de desempleo paterno y $18,2 \%$ de desempleo materno. La gran mayoría de los padres poseen el primer grado de escolaridad incompleto (80\%) y algunos son analfabetos (8\%). Un $21,4 \%$ de los adolescentes relatan la existencia de problemas referidos al consumo de alcohol en algún familiar que vive en el mismo hogar, y un $5,0 \%$ informa de problemas con el consumo de otras drogas en un miembro de la familia.

Sobre las relaciones familiares (Tabla 3), se observa que la mayoría $(55,8 \%)$ clasifica como 
Tabla 2

Quejas que motivaron la búsqueda de atención médica por parte de adolescentes.

Unidades de atención primaria municipales, centro urbano del sur de Brasil, 1997-1998.

\begin{tabular}{lrr}
\hline Motivos de consulta & $n$ & $\%$ \\
\hline Relacionados con el embarazo & 114 & 24,6 \\
Relacionados con el aparato respiratorio & 49 & 10,6 \\
Relacionados con quejas dermatológicas & 40 & 8,6 \\
Relacionados con el sistema osteomuscular & 38 & 8,2 \\
Relacionados con el aparato digestivo & 31 & 6,7 \\
Relacionados con quejas neurológicas & 25 & 5,4 \\
Solicitar o ver resultados de exámenes (citopatológico incluido) & 22 & 4,8 \\
Relacionados con quejas generales o inespecíficas & 21 & 4,5 \\
Relacionados con la menstruación con o sin otros síntomas & 18 & 3,9 \\
Relacionados con los genitales & 18 & 3,9 \\
Relacionados con las relaciones sexuales y/o anticoncepción & 17 & 3,7 \\
Solicitar certificado médico & 12 & 2,6 \\
Relacionados con el aparato urinario & 11 & 2,4 \\
Relacionados con múltiples órganos & 9 & 1,9 \\
Relacionados con el aparato cardiocirculatorio & 8 & 1,7 \\
Relacionados con la sangre y/u órganos hematopoiéticos & 7 & 1,5 \\
Relacionados con quejas oculares y/o auditivas & 7 & 1,5 \\
Relacionados con quejas psicosociales & 7 & 1,5 \\
Relacionados con las mamas & 6 & 1,3 \\
Relacionados con el sistema endócrino & 1 & 0,2 \\
Ignorados & 2 & 0,4 \\
Total & 463 & 100,0 \\
\hline
\end{tabular}

buena la calidad del relación parental. Menos frecuentes son las relaciones consideradas como muy conflictivas con agresión física (2,7\%). En cuanto a la calidad de la relación entre el adolescente y sus padres, la mayor parte $(71,2 \%)$ siente esa relación como buena y los menos la sienten como conflictiva y / o indiferente (16,3\%). Un $84,8 \%$ caracteriza la relación con su madre como buena y un $8,3 \%$ como conflictiva y / o indiferente.

Según la percepción del 90,3\% de los adolescentes, los padres de alguna manera refuerzan positivamente a los hijos cuando hacen algo que les parece bien principalmente con elogios $(45,6 \%)$ y, cuando hacen algo incorrecto, muchos de los padres intentan conversar con sus hijos (54,8\%). Sin embargo, el 30,6\% de los padres reaccionan con agresividad tanto psicológica (descalificando 13,3\%; culpabilizando $12,4 \%$; amenazando, 3,3\%) como física (a golpes $1,5 \%$ ). Se observa que la diferencia en la percepción de los adolescentes no es significativa según el sexo.

Según los jóvenes, en cuanto a la imposición de algunos límites por parte de los padres, se verifica que un $88,4 \%$ no establecen hora para que el adolescente se acueste, un $71,7 \%$ de los jóvenes tienen que decir siempre dónde van, cuándo salen y para un $48,6 \%$ de los adolescentes algunas cosas los padres se las permiten hacer otras no, y un $42,2 \%$ de los padres raramente les prohíben hacer algo. También, un 55,2\% de los adolescentes entrevistados creen que pueden ser escuchados por sus padres, o por lo menos por uno de ellos, siempre y cuando lo necesitan, mientras que un $26,7 \%$ sólo a veces y un $18,0 \%$ cree que no puede ser escuchado por sus padres. No se encontró diferencia entre los sexos.

Sobre el sentimiento de ser criticado por sus padres por su modo de ser o de vestirse, la mayoría de los jóvenes no se sienten criticados $(65,2 \%)$. No obstante, con la estratificación por sexo, se observa que las mujeres se sienten más criticadas que los varones ( $p \varangle 0,01$ ). Sobre el interés de los padres por el desempeño escolar de los hijos que viven con ellos, la mayoría se preocupan con el tema todo el año (82\%), pero se interesan más por los estudios de los hijos que por los de las hijas ( $p<0,001$ ).

Sobre la actividad que desarrollan, muchos adolescentes estudian $(45,8 \%)$, o ni estudian ni trabajan fuera de casa $(37,4 \%)$. Un $8,0 \%$ sólo 
Distribución de cómo los adolescente perciben la calidad de la relación familiar.

Unidades de atención primaria municipales, centro urbano del sur de Brasil, 1997-1998.

\begin{tabular}{|c|c|c|c|c|}
\hline Variables & $\mathrm{n}$ total1 & $\begin{array}{l}\% \text { del total } \\
\text { masculino }\end{array}$ & $\begin{array}{l}\% \text { del total } \\
\text { femenino }\end{array}$ & $\%$ total \\
\hline Calidad de la relación familiar & 407 & & & \\
\hline Buena & & 66,7 & 52,9 & 55,8 \\
\hline Regular & & 10,7 & 7,4 & 8,1 \\
\hline Conflictiva & & 4,8 & 6,8 & 6,4 \\
\hline Separados con relación cordial & & 6,0 & 11,5 & 10,3 \\
\hline Separados con relación tensa & & 3,6 & 7,4 & 6,6 \\
\hline No hay relación & & 8,3 & 13,9 & 12,8 \\
\hline Calidad de la relación con el padre & 393 & & & \\
\hline Buena & & 73,8 & 70,6 & 71,2 \\
\hline Regular & & 13,1 & 12,3 & 12,5 \\
\hline Conflictiva/indiferente & & 13,1 & 17,2 & 16,3 \\
\hline Calidad de la relación con la madre 2 & 446 & & & \\
\hline Buena & & 92,6 & 82,6 & 84,8 \\
\hline Regular & & 3,2 & 8,0 & 7,0 \\
\hline Conflictiva/indiferente & & 4,2 & 9,4 & 8,3 \\
\hline
\end{tabular}

1 Los totales no coinciden debido a la falta de información de algunos adolescentes

(p. ej. muerte de uno de los padres, no saber contestar).

2 Diferencia entre los sexos estadísticamente significativa $(p<0,01)$.

trabaja y un 8,9\% estudia y trabaja. La estratificación por sexo demuestra que muchas de las jóvenes de la muestra o sólo estudian (43,6\%) o ni estudian ni trabajan fuera del hogar (43,0\%), mientras la mayor parte de los varones estudia $(54,1 \%)$, y los que entran en la categoría "ni estudia ni trabaja fuera del hogar" corresponden a un $16,3 \%$. Al relacionarlo con la edad se verifica que a medida que aumenta la edad va disminuyendo el porcentaje de jóvenes que sólo estudian y aumentando aquellos que sólo trabajan o que estudian y trabajan, pero sobretodo aumentan las tasas de los que ni estudian ni trabajan fuera del hogar ( $p<0,001$ ).

Cuando se verifica la relación entre las variables actividad, estado civil y sexo llama la atención que de los 122 adolescentes que están casados o viviendo maritalmente $(26,3 \%)$, la mayoría significativa pertenece al sexo femenino (118 mujeres/ 4 varones) la mayor parte tiene edad comprendida entre los 18 y 19 años $(63,1 \%)$ y ni estudia ni trabaja fuera del hogar $(77,9 \%)$. Así, se comprende que las tasas de adolescentes tardíos que entran en la categoría "ni estudian ni trabajan fuera del hogar" está formada principalmente por las chicas casadas.

Sobre la frecuencia de algunas situaciones estresantes por las que han pasado los adoles- centes (Tabla 4) los hechos que más se produjeron fueron muerte de un ser querido que no era uno de los padres o hermanos (76,1\%), suspensión en la actividad escolar $(61,3 \%)$ y ruptura de relación amorosa (52,3\%). Respecto a cómo sienten que las mismas les han afectado, las hemos dispuesto en la tabla 4 en orden decreciente, según la referencia "afectó mucho". Así, se vio que los hechos que más afectaron a los jóvenes fueron la muertedela madre (94,1\%), muerte del padre $(84,5 \%)$ y enfermedad en familiar que vive en el mismo hogar $(76,4 \%)$. Vale poner en relevancia que entre los que han pasado por la situación de suspensión en el colegio de la actividad escolar, a la mayoría les afectó mucho $(54,9 \%)$ así como a aquellos que han dejado los estudios a muchos les ha afectado mucho $(46,6 \%)$, principalmente a las solteras. Las situaciones que más alegraron a los jóvenes fueron empezar a trabajar (62,9\%), adopción $(50,0 \%)$ y cuidar de hermanos o persona mayor $(37,8 \%)$.

\section{Discusión}

Algunas limitaciones del estudio necesitan ser consideradas. Por basarse en servicios de salud, los resultados no pueden ser extendidos a 
Tabla 4

O currencia de situaciones estresantes en la vida de los adolescentes y su referida repercusión.

Unidades de atención primaria municipales, centro urbano del sur de Brasil, 1997-1998.

\begin{tabular}{|c|c|c|c|c|c|c|}
\hline \multirow[t]{2}{*}{ Situaciones } & \multirow[t]{2}{*}{$\mathrm{n}$} & \multirow[t]{2}{*}{$\%$} & \multicolumn{4}{|c|}{ Repercusión referida (\%) } \\
\hline & & & No le afectó & Le afectó poco & Le afectó mucho & Le alegró \\
\hline Muerte de la madre & 17 & 3,7 & 0 & 5,9 & 94,1 & 0,0 \\
\hline Muerte del padre & 58 & 12,5 & 3,4 & 12,1 & 84,5 & 0,0 \\
\hline Enfermedad en familiar que vive en el hogar & 148 & 32,2 & 4,1 & 18,9 & 76,4 & 0,7 \\
\hline Enfermedad en otro pariente 0 amigo & 171 & 37,1 & 5,8 & 24,6 & 69,0 & 0,6 \\
\hline Violencia física & 67 & 14,5 & 9,0 & 23,9 & 67,2 & 0,0 \\
\hline Muerte de otro ser querido & 352 & 76,1 & 4,8 & 28,1 & 67,0 & 0,0 \\
\hline Suspensos en el colegio & 284 & 61,3 & 12,3 & 31,7 & 54,9 & 1,1 \\
\hline Mal desempeño en la escuela o trabajo & 208 & 44,9 & 12,0 & 37,0 & 50,5 & 0,5 \\
\hline Conflictos continuos con otras personas & 163 & 35,2 & 18,4 & 30,1 & 49,7 & 1,8 \\
\hline Muerte de hermano & 53 & 11,4 & 28,3 & 24,5 & 47,2 & 0,0 \\
\hline Dejó los estudios & 208 & 44,9 & 20,7 & 27,9 & 46,6 & 4,8 \\
\hline Ruptura de relación amorosa & 242 & 52,3 & 14,9 & 30,6 & 46,3 & 8,3 \\
\hline Pasó a vivir sin la familia & 88 & 19,0 & 13,6 & 33,0 & 39,8 & 13,6 \\
\hline Separación o divorcio de los padres & 148 & 32,5 & 24,3 & 31,1 & 39,2 & 5,4 \\
\hline Dificultad de relación en la escuela o trabajo & 105 & 22,7 & 16,2 & 45,7 & 38,1 & 0,0 \\
\hline Dificultades materiales & 162 & 35,0 & 22,2 & 43,8 & 33,3 & 0,6 \\
\hline Adopción & 14 & 3,0 & 14,3 & 14,3 & 21,4 & 50,0 \\
\hline Cambio de ciudad, escuela o barrio & 210 & 45,3 & 39,0 & 21,4 & 11,9 & 27,6 \\
\hline Cuidar de hermanos o persona mayor & 196 & 42,3 & 35,2 & 16,8 & 10,2 & 37,8 \\
\hline Empezó a trabajar & 159 & 34,3 & 18,9 & 10,1 & 8,2 & 62,9 \\
\hline
\end{tabular}

la población adolescente en general, pero sí entre aquellos que se presentan en unidades de atención primaria públicas de la zona urbana de comunidades semejantes. También, la manera como se realizó la encuesta (cara a cara) y constando la dirección del entrevistado seguramente perjudicó la veracidad de algunas respuestas, como el consumo de drogas y presencia de violencia familiar. También, pueden existir distorsiones en cuanto a la renta familiar per cápita, porque es posible que muchos de los adolescentes no tengan conocimiento de la verdadera remuneración de la familia. Sin embargo, los datos presentados tienen importancia fundamental, pues ponen en relevancia la realidad de los jóvenes que a menudo pasa desapercibida.

Según informe del PROSAD (MS, 2003a) muchas de las intervenciones fallan por adoptar un objetivo demasiado limitado e individualizado para un determinado problema de salud, sin preocuparse con la multiplicidad de factores que generan problemas en esa etapa de la vida. Así, algunas de las situaciones encontradas posiblemente reflejan aspectos del contexto sociocultural de la demanda que necesitan ser valorados y pensados como puntos de intervención, como puede ser el predominio de adolescentes del sexo femenino en la utilización de los servicios, el mayor porcentaje de mujeres entre los casados mostrando que el matrimonio y tener hijos fueron las situaciones que más les alegraron; la preponderancia de mujeres entre aquellos que se sienten más criticados por los padres y entre los que ni estudian ni trabajan fuera del hogar; y la observación de que dejar los estudios afectó menos a las chicas casadas que a las solteras. Por otro lado, la mayoría de los chicos estudian y algunos ya trabajan fuera de casa, y constatable el hecho de que los padres se interesen más por los estudios de los hijos que por los de las hijas.

Culturalmente, las mujeres suelen desarrollar un estilo menos activo de enfrentar las situaciones, se perciben ellas mismas como portadoras de menos recursos y eficacia que los hombres, son más introspectivas y suelen tener una valoración de sí mismas mucho más baja que ellos (Allgood-M erten et al., 1990). Esto puede influir a que estén más centradas en su cuerpo de un modo más orgánico o hipocondríaco (Weller, 1997, WHO, 1999) motivando la búsqueda de atención. La autoevaluación negativa puede presentarse como causa o con- 
secuencia del sentimiento de ser criticada por los padres, contribuyendo al aparición de trastornos depresivos con todo su abanico de consecuencias (problemas escolares, quejas somáticas, relaciones conflictivas con su entorno, y otros) (Palazzo et al., 2001; Weissman et al., 1999; Zwaigenbaum et al., 1999). El matrimonio parece ser un importante objetivo para las jóvenes posiblemente por darles un status social y una seguridad. También puede que, en algunas ocasiones, funcione como la única alternativa de porvenir o un medio para huir de casa en búsqueda de mejores condiciones de vida. En cualquiera de los casos, pasan valorar positivamente hechos como el matrimonio y tener hijos. Quizás estos aspectos estén en la base de algunos embarazos en esa etapa, Ilevando a que desempeñen el rol de mujeres adultas, en detrimento de otros más apropiados para su edad, como el estudio.

Los varones, a su vez, son estimulados a la objetividad y constructos de superioridad y dominio (Allgood-Merten et al., 1990). Son preparados para el trabajo fuera del hogar $y$, principalmente, en un medio socioeconómico bajo, su papel suele ser el de un proveedor. El mercado de trabajo ya ha absorbido a algunos, Io que agrada a muchos por insertarse en el contexto. Por otro lado, se verifica que raramente buscan los servicios asistenciales y cuando lo hacen suele ser principalmente en situaciones emergenciales existiendo por tanto poca oportunidad para acciones preventivas. Esto es preocupante, pues los varones suelen estar a menudo bajo riesgo de morbilidad y mortalidad, principalmente por lesiones, homicidios, suicidios, enfermedades sexualmente transmisibles y abuso de sustancias (Ziv et al., 1999).

El aumento del índice de utilización de los servicios a medida que aumenta la edad parece asociarse con un incremento en la autonomía para la búsqueda de atención. No obstante, teniendo en cuenta la frecuencia de diagnósticos como el embarazo, es posible que refleje también las secuelas de una conducta de riesgo en la adolescencia temprana.

El predominio de quejas dispersas en el campo somático y relacionadas a la sexualidad como motivo de contacto con los servicios, está de acuerdo con lo encontrado por otros autores (Colino et al., 1995; Fisher et al., 1988; Formigli et al., 2000) y pone de relevancia la importancia de estos aspectos en la adolescencia. El cuerpo es el medio a través del cual el joven se muestra a los demás y así se convierte en lo manifiesto. Sin embargo, las transformaciones sufridas pueden generar ansiedad, haciendo que él deposite sus angustias en una parte u órgano del cuerpo, y que se exprese a través de quejas físicas (Outeiral, 1994). En cuanto a la sexualidad, es en la adolescencia cuando se da la organización sexual definitiva representando, así, una de las tareas fundamentales de esta etapa (Marcelli \& Braconnier, 1989).

Llama la atención que la gran mayoría de los adolescentes no relató deseo de conversar con su médico sobre otros temas a no ser la queja principal, revelando casi nada sobre consumo de drogas, situaciones estresantes, conflictos familiares, abuso sexual y otros aspectos emocionales, aunque algunos de ellos han sufrido con este tipo de vivencias. Lo mismo se verifica respecto a temas vinculados a la educación, como puede ser la anticoncepción. Creemos que esta postura puede reflejar la concurrencia de varios factores, como aquellos relacionados a la cultura (pasividad), al modo como los servicios se presentan ante la comunidad (posiblemente más como curativos), a las creencias de los jóvenes de que es el médico la persona que tiene que proponer la conversación, la dificultad y vergüenza para hablar de temas personales (Steiner \& Gest, 1996), que el médico es un adulto lejano (Malus, 1987) o que las personas más indicadas para ayudarlos en sus problemas son los amigos y los padres (Offer et al., 1991); falta de conocimiento sobre la existencia de un tratamiento efectivo, indiferencia, ignorancia acerca de las condiciones de riesgo y sus consecuencias, y aspectos relacionados con la confidencialidad (Colino et al., 1993; Klein et al., 1998). Por otro lado, se observa que los médicos no van más allá de la queja principal lo que también puede ser el resultado de varios aspectos, como la escasez de tiempo en la consulta, la dificultad de lidiar con problemas del ámbito psicosocial o la poca importancia dada a estos elementos hasta el punto de no registrarlos debido, quizás, al estilo de formación médica que fomenta la dicotomía cuerpo/mente a través de un énfasis puesto sobre las enfermedades somáticas (Iacoponi, 1988; Steiner \& Gest, 1996; Ziv et al., 1999). Todo ello propicia a que muchas situaciones o comportamientos de riesgo pasen sin merecer la debida atención.

Aun así, las unidades, a pesar de las dificultades señaladas, parecen ser un punto de referencia, pues los adolescentes acuden a ellas cuando perciben algún problema de salud. Posiblemente colabora para eso, entre otros factores, el hecho de que los servicios, en general, atienden a las personas de la zona en que están ubicados, lo que facilita la accesibilidad. De cualquier forma, las unidades de atención pri- 
maria, con excepción de situaciones perinatales, son utilizados en el sentido más curativo que preventivo, reflejando pues la necesidad de implementación de programas como PSF y PACS para potenciar acciones de promoción y prevención de manera integral y continua.

Sobre el modo de vida de los adolescentes, la familia nuclear parece ser la base. En los casos en que hubo una reestructuración de la familia es escaso el relato de adolescentes que viven con el padre y su nueva compañera. Esto refleja el comportamiento habitual de las parejas que, en general, cuando hay separación o divorcio, es el hombre el que deja la casa y la mujer se queda con los hijos (Pujet \& Berenstein, 1994). Es interesante destacar que algunos jóvenes conviven en el mismo hogar con hermanastros, lo que supone que algunas estructuras familiares han absorbido hijos frutos de relaciones fuera de la estructura parental.

La relación parental es principalmente buena, con unas tasas muy bajas de relaciones conflictivas sobretodo acompañadas de agresión física. El hallazgo se contrapone a Fishman (1990) que relata que la incidencia de violencia física entre cónyuges está por encima del $16 \%$ en un período de un año y del $28 \%$ en el transcurso de la relación matrimonial. De igual modo, aunque muchos adolescentes han relatado que las relaciones con sus padres son buenas, es considerable el número de los que informan de reacciones agresivas físicas y psicológicas de éstos cuando los hijos hacen algo que les parece mal. En general, el abuso físico suele insertar-se en una situación más amplia de abandono resultando en sentimientos de soledad y desamor (Carpenter \& Treacher, 1993). La violencia psicológica es un maltrato oculto. Así, un joven constantemente despreciado y humillado ve impedido su adecuado desarrollo psicológico y de sus aptitudes.

Sobre las situaciones de estrés vividas, el relato de la muerte de uno de los padres es una de las situaciones que más afectó a los adolescentes. Es comprensible considerando que la madre y el padre son los personajes más importantes en el desarrollo de la personalidad del individuo (Bifulco et al., 1987). Enfermedad de un familiar que vive en el mismo hogar es la segunda situación que más afectó a los jóvenes y esta suele ser una situación muy difícil para todos los miembros de una familia, pues tiene como consecuencia la ruptura de la homeostasis y equilibrio de la familia, y un agotamiento físico y mental para algunos de sus miembros. Sin embargo, llama la atención que el hecho de cuidar hermanos o persona mayor fue una de las situaciones que más alegró a los adolescen- tes. Existen familias en las que, sea por fuerza de la necesidad o por el modo de estructuración del sistema familiar, hacen emerger la figura del hijo parentalizado en que el papel del adulto se coloca en su lugar a un hijo. Este tipo de experiencia suele ser una sobrecarga emocional. Así, esta "alegría” puede ser un mecanismo de defensa utilizado para ocultar probables sentimientos de rabia o tristeza vinculados a esta vivencia para la que el adolescente no está, ni cognitivamente ni psicológicamente, maduro para llevar a cabo pudiendo ocasionar problemas emocionales futuros (Bekir et al., 1993).

La escuela es un sitio especial de socialización, de desarrollo de la autoestima y habilidades personales. Por eso, un fallo en este ámbito (suspensos en el colegio, mal desempeño en la escuela y dejar los estudios) afecta a muchos de los jóvenes en estos aspectos.

Considerando lo expuesto, creemos que es esencial que los gestores de salud proporcionen entrenamiento para los profesionales de atención primaria, PSF y agentes comunitarios de salud en relación con la adolescencia y sus peculiaridades, estimulando a que puedan acercarse dónde ellos están, principalmente en relación a los adolescentes del sexo masculino. Además, es fundamental que sean entrenados a dar orientación a las familias para que los padres puedan adquirir habilidades y conocimientos suficientes sobre temas como sexualidad, enfermedades sexualmente transmisibles, anticoncepción, uso de drogas, relación familiar y otros, para que puedan orientar y promover una mejor salud para sus hijos. También, es importante desarrollar programas en escuelas, incluyendo a los profesores, para que puedan mejorar sus conocimientos sobre la adolescencia, permitiéndoles estimular en los jóvenes actitudes hacia la salud, ayudando así a los padres y alumnos.

Por fin, es importante fomentar estudios que puedan evaluar, en la cultura, el papel del profesional sanitario y las condiciones de trabajo en el contexto de la atención básica que puedan estar contribuyendo a la dificultad de lidiar con los problemas psicosociales de los adolescentes. También es esencial Ilevar a cabo estudios cualitativos con los adolescentes para que, a partir de una escucha sobre sus vivencias, expectativas y visión del mundo, se pueda comprender mejor cómo se expresan y buscan ayuda, con el objetivo de auxiliar y orientar a la creación de medidas más eficaces de abordaje, ayudando así a mejorar la calidad de la vida de este grupo de personas. 


\section{Agradecimiento}

Agradecimientos a la Secretaría de Salud y Bienestar de la ciudad de Pelotas, a través de la persona del entonces Secretario Municipal de Salud, Dr. Kleber Ramil, por haber permitido y dado condiciones a la realización del estudio.

\section{Referencias}

ALLGOOD-MERTEN, B.; LEWINSHON, P. M \& \& HOPS, H., 1990. Sex differences and adolescent depression. Journal of Abnormal Psychology, 99:55-63.

BEKIR, P.; M CLELLAN, T.; CHILDRESS, A. L. \& GARITI, P., 1993. Role reversal in families of substance misusers: A transgenerational phenomenon. Internal Journal of Addictions, 28:613-630.

BIFULCO, A.; BROWN, G. \& HARRIS, T., 1987. Childhood loss of a parent, lack of adequate parental care and adult depression: A replication. Journal of Affective Disorders, 12:115-128.

BLUM, R., 1987. Physicians assessment of deficiencies and desire for training in adolescent care. Journal of Medical Education, 62:401-407.

CARPENTER, J. \& TREACHER, A., 1993. Problemas y Soluciones en Terapia Familiar y de Pareja. BarceIona: Ediciones Paidós.

COLINO, N. R.; FERNANDEZ-BARRERO, A. \& MARTINEZ, P. U., 1995. Utilización de la consulta de atención primaria por los adolescentes y detección de problemas de salud no demandados. Atención Primaria, 16:594-600.

CURBELO, A. A., 1987 Demografía Sanitaria de la Adolescencia. Madrid: Ministerio de Sanidad.

FISHER, M.; MARKS, A. \& TRIELLER, K., 1988. Meeting the health care needs of suburban youth: Review of a clinical service. Pediatrics, 81:8-13.

FORMIGLI, V. L. A.; COSTA, M. C. O. \& PORTO, L. A., 2000. Avaliação de um serviço de atenção integral à saúde do adolescente. Cadernos de Saúde Pública, 16:831-841.

FISHM AN, H. C., 1990. Tratami ento de Adol escentes con Problemas. Un Enfoque de Terapia Familiar. Barcelona: Editorial Paidós.

IACOPONI, E., 1988. Metodological issues in measuring the detection of emocional disorders by primary care physicians - A review of the literature. Revista de Saúde Pública, 22:46-56.

KLEIN, J. D.; MacNULTY, M. \& FLATAU, C. N., 1998. Adolescents's access to care. Archives of Pediatric and Adolescent Medicine, 152:676-682.

MALUS, M.; LA CHANCE, P. A.; LAMY, L.; MacALAY, A. \& VANASSE, M., 1987. Priorities in adolescent health care: The teenager's viewpoint. Journal of Family Practionner, 25:159-162.

MARCELLI, D. \& BRACONNIER, A., 1989. Manual de Psi copatologia do Adol escente. Porto Alegre: Editora Artes Médicas.

MARKS, A.; FISHER, M. \& LASKER, S. 1990 .Adolescent medicine in pediatric practice. Journal of Adolescent Health Care 11:149-153.

MS (M inistério da Saúde), 2003a. Programa de Saúde do Adolescente (PROSAD). 2 Junho 2003 «ttp:// www.saude.org. br/sps/areastecnicas/ adolescente/ apresentaçao.htm>.
MS (Ministério da Saúde), 2003b. Programa de Saúde da Família e Agentes Comunitários de Saúde (PSF ePACS). 2 de Junho de 2003 ঝttp:// www.saude. org.br/psf/programa/index.asp>.

OFFER, D.; HOWARD, K. I.; SCHONERT, K. A. \& OSTROV, E., 1991. To whom do adolescents turn for help? Differences between disturbed and nondisturbed adolescents. Journal of American Academic of Child and Adolescent Psychiatry, 30:623-630.

OUTEIRAL, J. O., 1994. Adol escer. Estudos sobre Adolescência. Porto Alegre: Editora Artes Médicas.

PALAZZO, L. S.; BERIA, J. U.; ALONSO-FERNÁNDEZ, F. \& TOM ASI, E., 2001. Depresión en la adolescencia en centros de atención primaria: Importancia de un problema oculto en salud colectiva. Atención Primaria, 28:543-549.

PUJET, J. \& BERENSTEIN, I., 1994. Psicanálise do Casal. Porto Alegre: Editora Artes Médicas.

RUBIO, J. L., 1987. Adolescencia y Salud Mental. España: Ayuntamiento de L'Hospitalet de Llobregat.

STEINER, B. D. \& GEST, K., 1996. Do adolescents want to hear preventive counseling messages in outpatient settings? Journal of Family Practionner, 43:375-381.

TAVARES, B. F.; BERIA, J. U. \& LIM A, M. S., 2001. Prevalência do uso de drogas e desempenho escolar entre adolescentes. Revista de Saúde Pública, 35:150-158.

WALKER, Z. \& TOWNSEND, J., 1998. Promoting adolescent mental health in primary care: A review of the literature. Journal of Adol escence, 21:621-634.

WHO (World Health Organization), 1999. Programming for Adol escent Health and Devel opment Report. Technical Series 886-99. Geneva: WHO.

WEISSM AN, M. M.; WOLK, S.; GOLDSTEIN, R. B.; MOREAU, D.; ADAMS, P.; GRENWALD, S.; KLIER, C. M.; RYAN, N. D. \& DAHL, R. E., 1999. Depressed adolescents grown up. JAMA, 281:1707-1713.

WELLER, S. I. \& ORELLANA, L. C., 1997. Los jóvenes y la salud. Construcción de grupos en función de representaciones sobre salud y enfermedad. Cadernos de Saúde Pública, 13:397-404.

WONCA (World Organization of Family Doctors), 1986. International Classification of Process in Primary Care. Oxford: Oxford University Press.

ZIV, A.; BOULET, J. R. \& SLAP, G. B., 1999. Utilization of physician offices by adolescents in the United States. Pediatrics, 104:35-42.

ZWAIGENBAUM, R.; SZATMARI, P.; BOYLE, M. H. \& OFFORD, D. R., 1999. Highly somatizing young adolescents and the risk of depression. Pediatrics 103:1203-1209.

Recibido el 28 de Junio de 2002

Versión final presentada el 11 de Junio de 2003

Aprobado el 14 de julho de 2003 